\title{
Guest Editorial: Special Issue on Experimentation in Economics
}

\author{
Hans M. Amman ${ }^{1} \cdot$ Marco P. Tucci $^{2}$ \\ Published online: 7 August 2020 \\ (c) Springer Science+Business Media, LLC, part of Springer Nature 2020
}

Experimentation methods are relevant in many strands of economic literature. This special issue presents some recent and original applications of these methods in economics. The goal is both to highlight topical research areas and to underline the interest of $\mathrm{CE}$ in this important area.

In "Heterogeneous expectations and uncertain inflation target" Stefano Marzioni and Guido Traficante analyze a new Keynesian economy populated by adaptivelearning agents with heterogeneous beliefs about the time varying inflation target. A fraction of agents is assumed to have a full and updated information set including the permanent and temporary component of the inflation target at the current period, while the remainder of agents receives a signal and use it to estimate the target components solving a Kalman filter problem. The proportion of the two strategies is endogenous and depends on a measure of past performance of predictors. They conduct stochastic simulations to assess whether different hypotheses about the information regime may affect macroeconomic stability in the short and in the long run.

Annarita Colasante, Simone Alfarano and Eva Camacho-Cuena, in "Heuristic Switching Model and Exploration-Exploitation Algorithm to describe long-run expectations in LtFEs: a comparison", compare the performance of two learning algorithms in replicating individual short and long-run expectations: the Exploration-Exploitation Algorithm (EEA) and the Heuristic Switching Model (HSM). To do so they implement the EEA proposed by one of the authors in a previous work and modify the existing version of the HSM in order to incorporate the long-run predictions. Individual expectations are elicited in a series of Learning-to-Forecast Experiments (LtFEs) with different feedback mechanisms between expectations and market price: positive and negative feedback markets.

Marco P. Tucci

tucci@unisi.it

Hans M. Amman

amman@uva.nl

1 Faculty of Economics and Business, University of Amsterdam, Roetersstraat 11, 1018 WB Amsterdam, The Netherlands

2 Dipt. di Economia Politica, Università di Siena, Piazza S. Francesco, 7, 53100 Siena, Italy 
An optimal control problem of a small nonlinear econometric model with uncertain parameters and passive learning (open-loop feedback) is considered in the paper by Dmitri Blueschke, Ivan Savin and Viktoria Blueschke-Nikolaeva entitled "An evolutionary approach to passive learning in optimal control problems." Following an approach proposed by two of the authors in a previous work, parameter uncertainty is here explicitly addressed with a large Monte Carlo experiment of possible parameter realizations and the optimization is carried out with the Differential Evolution algorithm. This method is extended to the case of passive learning and applied to a small dynamic model of the Austrian economy.

In "How Active is Active Learning: Value Function Method Versus an Approximation Method" Hans M. Amman and Marco P. Tucci compare the two dominant approaches for solving models with optimal experimentation (also called active learning), i.e. the value function and the approximation method. They extend the results of a previous paper to a version of Beck and Wieland ( $\mathrm{J}$ Econ Dyn Control 26:1359-1377, 2002) model with a stationary process and a positive penalty on the control. The two methods are then used to solve the same problem and their solutions are compared. To do so a new value function algorithm has been written, to handle several sets of parameters, and more general formulae for the cost-to-go function of the approximation method are used.

The last two works in this Special Issue present alternative algorithms applied to similar problems and allow some sort of methods comparison. In this respect they represent an ideal continuation of the "methods comparison project" initiated at the Washington D.C. meeting of the Society for Computational Economics in 2005 by Thomas Cosimano, Volker Wieland and David Kendrick. The "methods" under study were those used for learning and control, optimal experimentation and adaptive or dual control. In essence, methods for dynamic stochastic models in which the control variables can be used not only to guide the system in desired directions but also to improve the accuracy of estimates of parameters in the models. The purpose of this "project" was to better understand the comparative advantage of the three methods in their application to dynamic stochastic economic models.

The editor hopes that this Special Issue will illustrate to CE readers some of the most interesting recent advances in this area and will stimulate them to build upon the results reported in these pages. Finally, a sincere thanks goes to all the authors and referees for their cooperation and Naveen Parthiban and Kasturi Thilagam for their valuable assistance.

Publisher's Note Springer Nature remains neutral with regard to jurisdictional claims in published maps and institutional affiliations. 